\title{
Whole Transcriptome Analysis with Ion Torrent Platform on Challenging Tissues: Formalin-Fixed, Paraffin-Embedded Tissues and Laser Capture Microdissected Samples
}

Francesca Lessi ${ }^{1 *}$, Paolo Aretini ${ }^{1}$, Sara Franceschi ${ }^{1}$, Marco La Ferla ${ }^{1}$, Samuele Negro², Generoso Bevilacqua ${ }^{1}$ and Chiara Maria Mazzanti ${ }^{1}$

${ }^{1}$ Genomic Section, Pisa Science Foundation ONLUS, Via Panfilo Castaldi, 2, 56121, Pisa (PI), Italy

${ }^{2}$ Department of Biomedical Sciences, University of Padova, Italy

\begin{abstract}
Background: The advent of the NGS revolutionized cancer research by making it possible to study the complexity of cancer using high throughput sequencing methodologies. The current trends are to adapt highthroughput sequencing technologies to the level of small cell populations and even individual cells. In our laboratory, we developed some different methods that would allow us to work optimally with very low amount of material, based on the technique of the SMARTer technology and on the lon Torrent protocols that we modified carefully, applied to Ion Proton system.
\end{abstract}

Materials and methods: We collected 12 formalin-fixed, paraffin-embedded tissues and 20 laser capture microdissected formalin-fixed, paraffin-embedded samples derived from brain and breast cancer and 13 laser capture microdissected fresh frozen samples derived from mouse brain cells.

Results: We developed high performance methods to analyse the whole transcriptomes of our samples, obtaining a very high number of reads $(78,186,377$ usable reads), perfectly comparable with samples with a large amount of RNA such as samples obtained from cells or fresh tissues.

Conclusions: We have found that the combination of SMARTer technology and Ion TargetSeq Exome Enrichment kit, in addition to some improvements to their conventional protocols, provides excellent results with challenging samples.

Keywords: Transcriptome; Microdissection; Paraffin; Degraded material; Breast cancer; Tumour progression

\section{Introduction}

Formalin-fixed, paraffin-embedded (FFPE) tissue samples stored in diagnostic pathology archives represent an invaluable bio-bank for retrospective clinical research. This interest is primarily driven by the fact that the process of creating FFPE tissue is the most common technique used by clinical and/or research pathologists for tissue processing, evaluation, diagnostics, immunoanalysis, preservation, and archiving.

The use of FFPE samples in molecular studies presents some great advantages. For example, these types of samples are available and readily accessible in vast quantities. The cost associated with their storage is low, as well, and the significant association between pathological and clinical annotations makes FFPE tissue an attractive specimen for biomarker discovery. Nevertheless, as it is well known, this method of preservation causes chemical modification and degradation of nucleic acids. As a matter of fact, when working with nucleic acids, FFPE samples present some drawbacks as RNA and DNA can be degraded and modified by the fixation process and, independently, degraded with time.

Fortunately, the modern technologies have made it increasingly possible to work with this type of material, because of its great potential. Many solid cancers are characterized by heterogeneity within the cell populations. Cancer inter-tumor and intra-tumor heterogeneity is identified daily by pathologists and may have important consequences for personalized-medicine approaches. In fact, tissue specimens typically resected from cancer patients are composed of complex cell types including normal and malignant epithelial cells, fibroblasts, immune cells, and endothelial cells. Given the potential contributions of each cell type to tumor progression, the study of the tumor heterogeneity becomes more necessary [1].
Laser capture microdissection (LCM) allows to capture a population of homogeneous target cells from a heterogeneous tissue section. The method thus significantly improves the accuracy of the cell-specific molecular profiling, as it eliminates noise derived from cell contamination. The development of reliable NGS-based methods for use with low-quality FFPE tissue-derived nucleic acids (such as those derived from LCM FFPE tissues) would open the diagnostic pathology archives to high-throughput profiling, facilitating extensive retrospective clinical studies. In particular, RNA-Seq analysis allows to evaluate the whole transcriptome sequence for each gene; this is extremely important in FFPE tissue gene expression analysis which could lead to a lack of information using conventional techniques (e.g. Real-Time PCR) due to the non-uniformity of the sequence.

The current trends are to adapt high-throughput sequencing technologies to the level of small cell populations and even individual cells [2,3]. Analyses of transcriptomes through massively parallel sequencing of cDNAs (mRNA-Seq) generates millions of short sequence fragments that can be analyzed to accurately quantify expression levels, assemble new transcripts and investigate alternative

*Corresponding author: Francesca Lessi, Staff Scientist, Genomic Section, Pisa Science Foundation ONLUS, Via Panfilo Castaldi, 2, 56121, Pisa (PI), Italy, Tel: +39050974061; Fax: +390509656178; E-mail: f.lessi@fondazionepisascienza.org

Received June 09, 2016; Accepted October 14, 2016; Published October 16 , 2016

Citation: Lessi F, Aretini P, Franceschi S, La Ferla M, Negro S, et al. (2016) Whole Transcriptome Analysis with Ion Torrent Platform on Challenging Tissues: Formalin-Fixed, Paraffin-Embedded Tissues and Laser Capture Microdissected Samples. Next Generat Sequenc \& Applic 3: 136. doi: 10.4172/2469-9853.1000136

Copyright: ( $\odot 2016$ Lessi F, et al. This is an open-access article distributed under the terms of the Creative Commons Attribution License, which permits unrestricted use, distribution, and reproduction in any medium, provided the original author and source are credited. 
Citation: Lessi F, Aretini P, Franceschi S, La Ferla M, Negro S, et al. (2016) Whole Transcriptome Analysis with lon Torrent Platform on Challenging Tissues: Formalin-Fixed, Paraffin-Embedded Tissues and Laser Capture Microdissected Samples. Next Generat Sequenc \& Applic 3: 136. doi: 10.4172/2469-9853.1000136

Page 2 of 7

RNA processing. These techniques have been consistently pushed towards development of methods that require lower starting amounts of RNA, ideally down to single cells.

During the past few years, several groups have developed new sequencing-based methods for (single-cell) low input material transcriptome analysis but no one with Ion Proton platform, except Moroz et al. but their works are based only on fresh samples [4-7]. Furthermore, the are no studies in literature done with Ion Proton platform and LCM samples.

In our laboratory, we developed some different methods that would allow us to work optimally with very low amount of material, based on the technique of the SMARTer technology and on the Ion Torrent protocols that we modified carefully. This technology refined reverse transcription (RT), template switching and pre-amplification to obtain an increased cDNA yield from a very low input of cells, but with high sensitivity and less variability. In this paper, we perfected a method to analyze the whole transcriptome of different samples: FFPE, fresh and FFPE LCM samples on the Ion Proton Sequencer System (Life Technologies, Grand Island, NY).

\section{Materials and Methods}

\section{Samples}

12 FFPE tissues derived from glioblastoma tumor were chosen. For each sample 2 sections $10 \mathrm{~m}$ thick were cut using a new microtome blade for each tissue block and the material was collected in a $1.5 \mathrm{ml}$ tube (Table 1). 20 LCM FFPE samples derived from breast cancer some tumors (mainly breast cancer) were collected. We selected epithelial and stromal cells (from a maximum of 700 cells to a minimum of 100 cells) (Table 1). Moreover we selected 13 LCM fresh frozen samples derived from mouse brain cells (up to 20 cells) (Table 1).

\section{Laser capture microdissection}

Sections $2 \mathrm{~m}$ thick were cut from each case using a new microtome blade for each slide. The PALM RoboMover automatic laser microdissector (Carl Zeiss, Oberkochen, Germany) was used to select the epithelial and stromal cell population, inflammatory cells were carefully excluded.

\section{RNA isolation}

To isolate RNA from LCM samples we performed an incubation with 50 ul of lyisis buffer PKD (Qiagen, Venlo, Netherlands) and $10 \mathrm{ul}$ of proteinase K (Promega, Madison, WI, USA) at $55^{\circ} \mathrm{C}$ for overnight with the sample upside down. The day after we centrifuged the samples for 10 minutes at $10000 \mathrm{rpm}$ and we extracted RNA with the automated system Maxwell 16 (Promega, Madison, WI, USA) using the Maxwell ${ }^{\circ}$ 16 LEV RNA FFPE purification kit; we followed the protocol starting from the DNase treatment. For FFPE samples not microdissected we isolated RNA with the Maxwell ${ }^{\circ} 16$ LEV RNA FFPE purification kit following exactly the manufacturer's protocol. We measured RNA concentration of the FFPE samples using Qubit 2.0 fluorometer (Invitrogen, Life Technologies, Grand Island, NY) with the Qubit RNA HS assay kit or Qubit RNA assay kit according to the quantity of the starting material. We were not able to measure the concentration of the RNA extracted from LMD samples, even with Qubit RNA HS assay kit, because of the low amount of material.

\section{Library preparation}

The preparation of the library represents the susceptible part of our protocols and we applied different devices according to the type of samples that we had treated. For this reason, we explain the library preparation protocol divided into two sections:

- FFPE samples

- Fresh frozen and FFPE LCM samples

A) FFPE samples

\section{Ribosomal RNA removal}

rRNA was removed from our samples using the RiboGone mammalian kit (Clontech Laboratories, Takara Bio Inc., Mountain View, CA) starting from $50 \mathrm{ng}$ to $100 \mathrm{ng}$ of total RNA depending by the concentration obtained after RNA extraction.

\section{cDNA Synthesis}

To prepare cDNA from our RNA samples, we used the SMARTer Universal Low Input RNA kit (Clontech Laboratories, Takara Bio Inc., Mountain View, CA) that allows high-quality cDNA synthesis starting from as little as $200 \mathrm{pg}$ of input RNA. This kit has been validated for analysis with next-generation sequencing instruments just to produce NGS-quality cDNA from low concentrations of degraded samples [8]. We followed the guidelines of the protocol provided with the kit. The quality of our cDNA amplified was assessed using the Agilent 2100 bioanalyzer (Agilent Technologies, Santa Clara, CA), loading $1 \mathrm{ul}$ on the high sensitivity DNA chip from high sensitivity DNA kit (Agilent Technologies).

\section{Adapters ligation, size selection and amplification}

With these samples we avoided the fragmentation step because of our RNA was already quite fragmented being FFPE samples. The adapters were ligated to our cDNA using the Ion TargetSeq Exome Enrichment kit (Life Technologies). The samples were purified with Agencourt AMPure XP reagent (Beckman Coulter, Indianapolis, IN).

Our unamplified libraries, prepared and purified were run on the E-Gel SizeSelect 2\% agarose gel (Life Technologies) to obtain about 270 bp library DNA. The obtained bands were purified with Agencourt AMPure XP Reagent. The size-selected fragment library was then amplified with Platinum PCR SuperMix high fidelity (Life Technologies) with this PCR cycling program: $95^{\circ} \mathrm{C}$ for 15 seconds of denaturation; $95^{\circ} \mathrm{C}$ for 15 seconds, $58^{\circ} \mathrm{C}$ for 15 seconds, $70^{\circ} \mathrm{C}$ for 1 minute $\left(22\right.$ cycles) and $70^{\circ} \mathrm{C}$ for 5 minutes. After a new cycle of purification with Agencourt AMPure beads, the libraries were finally ready and we checked their quality with Agilent 2100 bioanalyzer using the high sensitivity DNA kit.

\section{B) Fresh and FFPE LCM samples}

\section{Ribosomal RNA removal}

Because of the insufficient amount of material, the total RNA was processed to cDNA, it was impossible to remove the rRNA. This was done at the time of data analysis, using appropriate filters.

\section{cDNA synthesis}

To prepare the library we used the SMARTer universal low input RNA kit as previously described. We can't assessed the quality of our cDNA libraries with Agilent 2100 bioanalyzer instrument because of the low quantity of the starting material, so we can't see anything after the run. Anyway we continued with our protocol.

\section{Adapters ligation, size selection and amplification}

Only the samples derived from fresh tissues were fragmented with 
Citation: Lessi F, Aretini P, Franceschi S, La Ferla M, Negro S, et al. (2016) Whole Transcriptome Analysis with lon Torrent Platform on Challenging Tissues: Formalin-Fixed, Paraffin-Embedded Tissues and Laser Capture Microdissected Samples. Next Generat Sequenc \& Applic 3: 136. doi: 10.4172/2469-9853.1000136

Page 3 of 7

\begin{tabular}{|c|c|c|c|c|c|}
\hline Samples & Organism & Type of sample & Tissue type & Year & Number of microdissected cells \\
\hline$\# 1$ & Homo sapiens & FFPE & Glioblastoma & 2010 & \\
\hline \#2 & Homo sapiens & FFPE & Glioblastoma & 2009 & \\
\hline$\# 3$ & Homo sapiens & FFPE & Glioblastoma & 2012 & \\
\hline$\# 4$ & Homo sapiens & FFPE & Glioblastoma & 2011 & \\
\hline$\# 5$ & Homo sapiens & FFPE & Glioblastoma & 2007 & \\
\hline \# 6 & Homo sapiens & FFPE & Glioblastoma & 2007 & \\
\hline$\# 7$ & Homo sapiens & FFPE & Glioblastoma & 2011 & \\
\hline$\# 8$ & Homo sapiens & FFPE & Glioblastoma & 2007 & \\
\hline$\# 9$ & Homo sapiens & FFPE & Glioblastoma & 2010 & \\
\hline$\# 10$ & Homo sapiens & FFPE & Glioblastoma & 2008 & \\
\hline \#11 & Homo sapiens & FFPE & Glioblastoma & 2008 & \\
\hline$\# 12$ & Homo sapiens & FFPE & Glioblastoma & 2009 & \\
\hline \# 13 & Homo sapiens & LCM FFPE & Ductal breast carcinoma & 2007 & 300 \\
\hline$\# 14$ & Homo sapiens & LCM FFPE & Ductal breast carcinoma & 2013 & 345 \\
\hline \# 15 & Homo sapiens & LCM FFPE & Ductal breast carcinoma & 2013 & 370 \\
\hline$\# 16$ & Homo sapiens & LCM FFPE & Ductal breast carcinoma & 2008 & 320 \\
\hline \# 17 & Homo sapiens & LCM FFPE & Ductal breast carcinoma & 2014 & 400 \\
\hline \# 18 & Homo sapiens & LCM FFPE & Ductal breast carcinoma & 2014 & 300 \\
\hline \#19 & Homo sapiens & LCM FFPE & Ductal breast carcinoma & 2009 & 430 \\
\hline$\# 20$ & Homo sapiens & LCM FFPE & Ductal breast carcinoma & 2010 & 260 \\
\hline$\# 21$ & Homo sapiens & LCM FFPE & Ductal breast carcinoma & 2010 & 330 \\
\hline$\# 22$ & Homo sapiens & LCM FFPE & Ductal breast carcinoma & 2012 & 380 \\
\hline$\# 23$ & Homo sapiens & LCM FFPE & Ductal breast carcinoma & 2010 & 410 \\
\hline$\# 24$ & Homo sapiens & LCM FFPE & Ductal breast carcinoma & 2010 & 250 \\
\hline$\# 25$ & Homo sapiens & LCM FFPE & Ductal breast carcinoma & 2011 & 310 \\
\hline$\# 26$ & Homo sapiens & LCM FFPE & Ductal breast carcinoma & 2013 & 350 \\
\hline$\# 27$ & Homo sapiens & LCM FFPE & Ductal breast carcinoma & 2009 & 330 \\
\hline$\# 28$ & Homo sapiens & LCM FFPE & Ductal breast carcinoma & 2011 & 320 \\
\hline$\# 29$ & Homo sapiens & LCM FFPE & Ductal breast carcinoma & 2011 & 385 \\
\hline \#30 & Homo sapiens & LCM FFPE & Ductal breast carcinoma & 2012 & 350 \\
\hline$\# 31$ & Homo sapiens & LCM FFPE & Ductal breast carcinoma & 2011 & 300 \\
\hline \# 32 & Homo sapiens & LCM FFPE & Ductal breast carcinoma & 2011 & 365 \\
\hline$\# 33$ & Mus musculus & LCM fresh frozen samples & Neuromuscolar junctions & & 20 \\
\hline \# 34 & Mus musculus & LCM fresh frozen samples & Neuromuscolar junctions & & 50 \\
\hline \# 35 & Mus musculus & LCM fresh frozen samples & Neuromuscolar junctions & & 50 \\
\hline \# 36 & Mus musculus & LCM fresh frozen samples & Neuromuscolar junctions & & 20 \\
\hline \# 37 & Mus musculus & LCM fresh frozen samples & Neuromuscolar junctions & & 20 \\
\hline \#38 & Mus musculus & LCM fresh frozen samples & Neuromuscolar junctions & & 50 \\
\hline \#39 & Mus musculus & LCM fresh frozen samples & Neuromuscolar junctions & & 50 \\
\hline$\# 40$ & Mus musculus & LCM fresh frozen samples & Neuromuscolar junctions & & 50 \\
\hline$\# 41$ & Mus musculus & LCM fresh frozen samples & Neuromuscolar junctions & & 50 \\
\hline$\# 42$ & Mus musculus & LCM fresh frozen samples & Neuromuscolar junctions & & 50 \\
\hline \# 43 & Mus musculus & LCM fresh frozen samples & Neuromuscolar junctions & & 50 \\
\hline \# 44 & Mus musculus & LCM fresh frozen samples & Neuromuscolar junctions & & 100 \\
\hline$\# 45$ & Mus musculus & LCM fresh frozen samples & Neuromuscolar junctions & & 100 \\
\hline
\end{tabular}

Table 1: Sample preparation of 12 FFPE tissues derived from glioblastoma tumor, 20 LCM FFPE derived from ductal breast cancer some tumors (mainly breast cancer) and 13 LCM fresh frozen samples derived from mouse brain cells.

Ion TargetSeq Exome Enrichment kit, in detail using the Ion Shear Plus Reagents for $3-5$ minutes at $37^{\circ} \mathrm{C}$ depending by the sample. For the FFPE samples instead we didn't fragment our cDNA because these are already degraded and fragmented. We performed the ligation of the adapters and of the barcodes (we loaded two libraries together in the same chip doing a pooled library) to the cDNA samples. For this part we followed the protocol of the Ion TargetSeq Exome Enrichment (Life Technologies). The samples were purified with Agencourt AMPure XP reagent (Beckman Coulter, Indianapolis, IN).

Our unamplified libraries, prepared and purified were run on the E-Gel SizeSelect 2\% Agarose Gel (Life Technologies, Grand Island, NY) to obtain about 270 bp library DNA. We didn't see the band of interest on the E-gel, because of the low starting material but thanks to the marker that run together with the samples, we could obtain the band at the right length. The size-selected fragment library was then amplified with Platinum PCR SuperMix high fidelity (Life Technologies, Grand Island, NY) with this PCR cycling program: $95^{\circ} \mathrm{C}$ for 15 seconds of denaturation; $95^{\circ} \mathrm{C}$ for 15 seconds, $58^{\circ} \mathrm{C}$ for 15 seconds, $70^{\circ} \mathrm{C}$ for 1 minute $\left(22\right.$ cycles) and $70^{\circ} \mathrm{C}$ for 5 minutes. and purified. The quality and the quantity of the amplified library was checked with Agilent 2100 bioanalyzer instrument with the Agilent high sensitivity DNA Kit.
Our libraries were ready for template preparation in Ion OneTouch 2 Instrument (Ion Torrent, Life Technologies, Grand Island, NY).

\section{Template preparation}

cDNA produced cannot be sequenced directly but amplification steps are required using emulsion PCR done with Ion OneTouch 2 instrument (Ion Torrent, Life Technologies, Grand Island, NY) following exactly the manufacturer's protocol. The non-templated ISPs were removed from the template ISPs with the Ion OneTouch ES instrument (Ion Torrent, Life Technologies, Grand Island, NY) following exactly the manufacturer's protocol.

\section{Sequencing on the Ion Proton}

To sequence our samples, we used Ion PI sequencing 200 kit (Ion Torrent, Life Technologies, Grand Island, NY). The Ion PI Chip (Ion Torrent, Life Technologies, Grand Island, NY) was prepared and calibrated for loading. We loaded the Ion PI Chip (Ion Torrent, Life Technologies, Grand Island, NY) with our template-positive ISPs and we run on Ion Proton sequencer.

\section{Bioinformatic analysis}

Bioinformatics analysis was carried out using several command 

Tissues: Formalin-Fixed, Paraffin-Embedded Tissues and Laser Capture Microdissected Samples. Next Generat Sequenc \& Applic 3: 136. doi: 10.4172/2469-9853.1000136

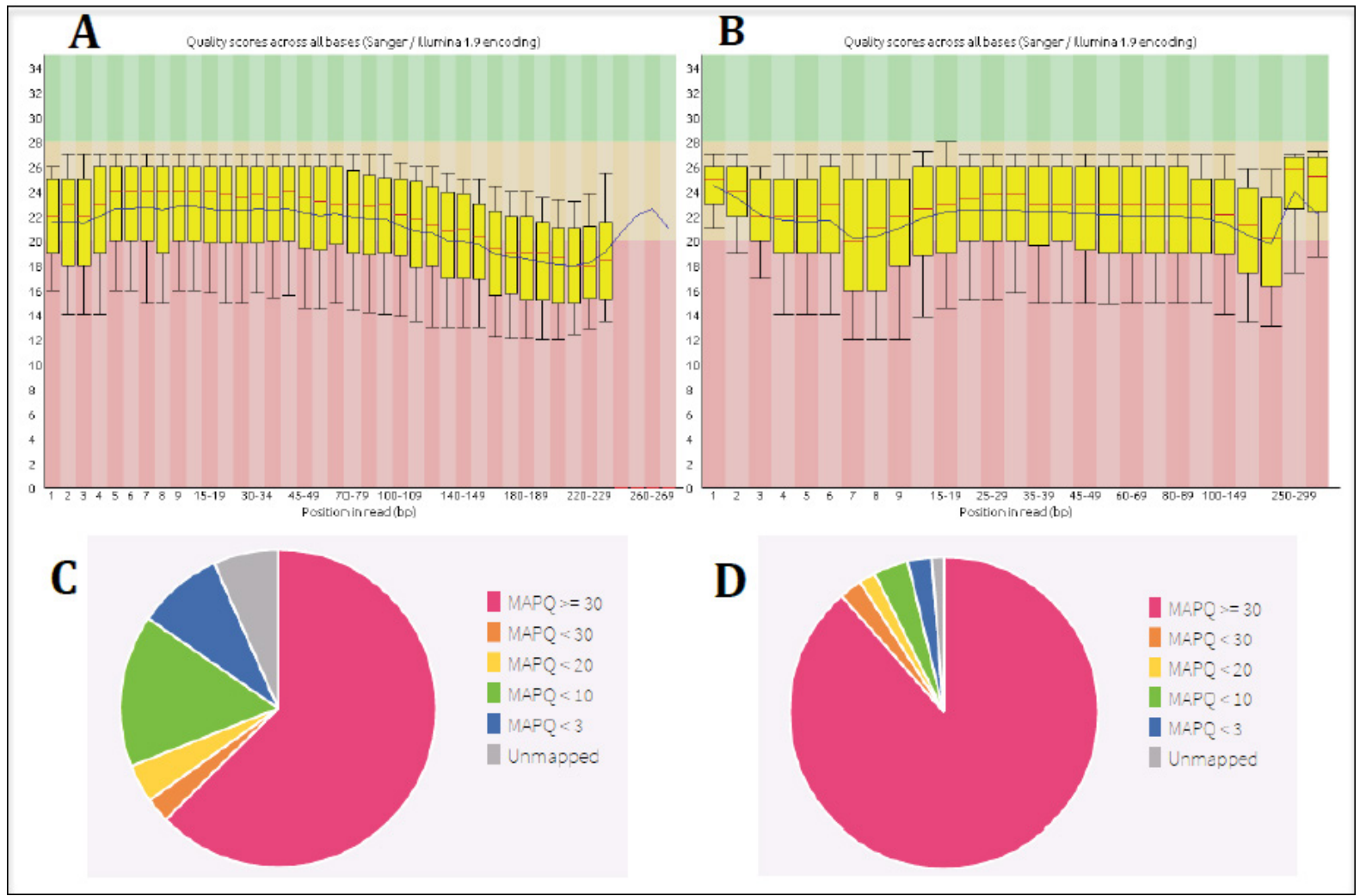

Figure 1: Quality score originated from FastgC, (A) FFPE samples done with lon Total RNA-seq kit (Life Technologies) and (B) FFPE samples done with our new protocol; Mapping quality obtained after the de-contamination, (C) FFPE samples done with lon Total RNA-seq kit and (D) FFPE samples done with our new protocol.

line software included in Bio-Linux (http://nebc.nerc.ac.uk/tools/ bio-linux/bio-linux-7-info) a custom version of Ubuntu 12.04 LTS. Fastq files were extracted from the Torrent Server and previously analysed for quality, length and presence of contaminants, by using the software Fastqc (http://www.bioinformatics.babraham.ac.uk/projects/ fastqc/) and Fastq Screen (http://www.bioinformatics.babraham. ac.uk/projects/fastq_screen/). Using star Aligner, the reads, previously filtered for the quality, length and contaminants, were processed and aligned to $H$. sapiens reference genome (build hg19) The unmapped reads, generated from the first step, were re-aligned by using Bowtie2 with the preset options "local" and "very sensitive" selected. The reads mapped with Star and Bowtie2 were then merged by using the Picard command SamMerge. The quality of the mapping was evaluated by using the SamStat software $[9,10]$.

\section{Results and Discussion}

Transcriptome analysis has further enabled our ability to reveal functionally relevant events in cancer research but unfortunately conventional RNA-Seq using low-input and archived material typically results in suboptimal performance. We developed high performance method to analyze the whole transcriptoms of our FFPE and LCM samples, obtaining a very high number of reads (with a maximum of 78186377 usable reads), perfectly comparable with samples with a large amount of RNA such as samples obtained from cells or fresh tissues. Ion Torrent technology offers kits to analyse the expression of genes of interest using custom or commercial panels (AmpliSeq ${ }^{\text {Tim }}$ Transcriptome Human Gene Expression Kit) or the whole transcriptome for fresh and frozen tissues, blood, cell culture and also for FFPE tissues (Ion Total RNA-Seq Kit). But unfortunately for FFPE tissues the results that we obtained, using this commercial kit following exactly their protocol, weren't optimal like those obtained with other types of samples (fresh, frozen). The quality score across all bases of the raw reads is not optimal as showed from plots originated from FastqC analyses (Figure 1A and 1B). Moreover, after the de-contamination the mapping quality too is worse in samples from commercial kits (Figure 1C and 1D) than in the samples made with our protocol.

We achieved a lot of reads but we had a non-uniformity of the sequence coverage (Figure $2 \mathrm{~A}$ ), the exons were partially covered by the reads in FFPE tissue compared to fresh tissue (Figure 2B). Instead with our modified protocol, the coverage become exactly comparable to samples of good quality, dispelling the myth of the bad success of the FFPE samples (Table 2). With these results we demonstrated that is possible to work with FFPE samples (Figure 3A) and that we can obtain very good results from these samples like we work with fresh tissues (Figure 3B). Another critical point in transcriptome analysis of clinically meaningful samples is the ability to sequence the limited quantities of material extracted from LCM samples. Also with these samples, we obtained a high coverage and a high read depth applying our modified protocol (Figure 3C and 3D). We overcome this obstacle using the SMARTer Universal Low Input RNA kit (Clontech) for the synthesis of cDNA and its amplification.

We have found that the combination of SMARTer Universal Low Input RNA kit and Ion TargetSeq Exome Enrichment kit, in addition to some improvements to their conventional protocols, provides to obtain excellent results and so analyze the whole transcriptome of different types of challenging samples. This goal makes it possible the use of the material stored in paraffin, as we know very common in the archives of all the pathological anatomies of the world. Indeed, this 
Citation: Lessi F, Aretini P, Franceschi S, La Ferla M, Negro S, et al. (2016) Whole Transcriptome Analysis with lon Torrent Platform on Challenging Tissues: Formalin-Fixed, Paraffin-Embedded Tissues and Laser Capture Microdissected Samples. Next Generat Sequenc \& Applic 3: 136. doi: 10.4172/2469-9853.1000136

Page 5 of 7

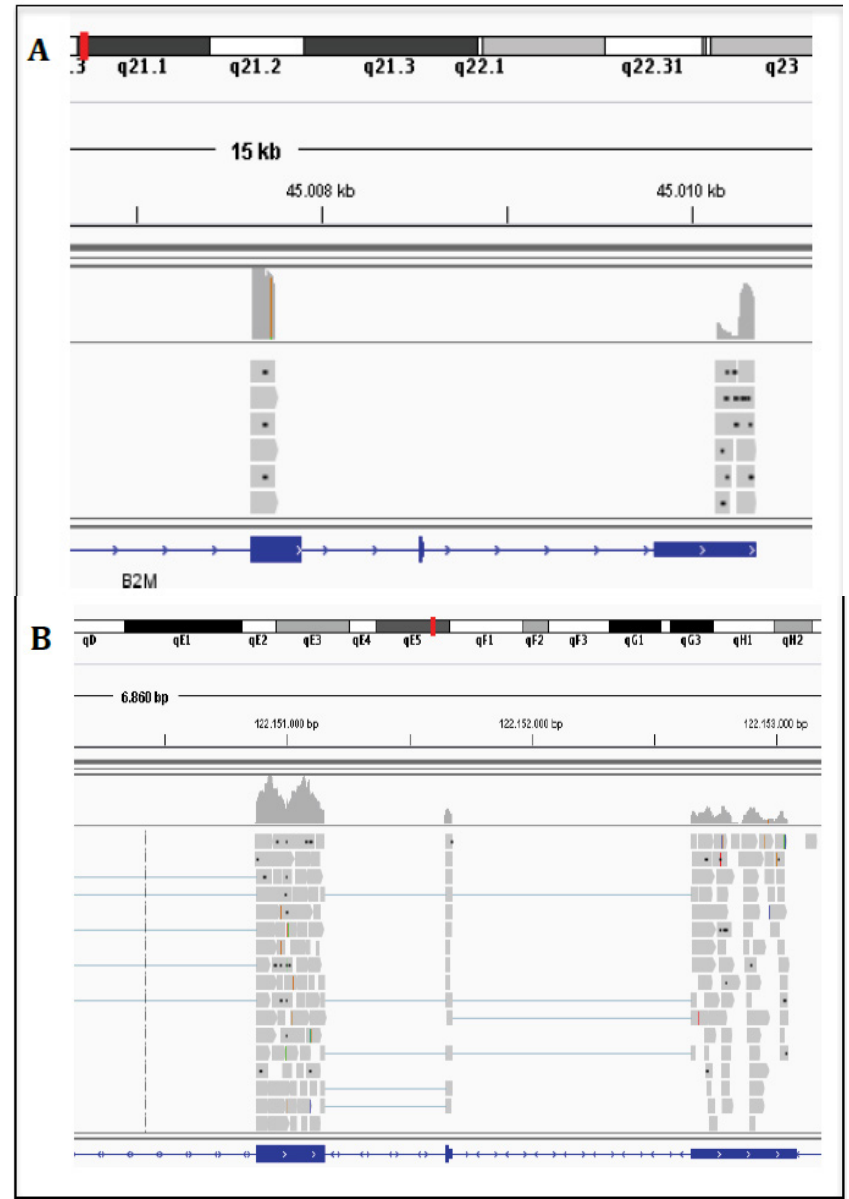

Figure 2: (A) FFPE sample made with lon Total RNA-seq kit (Life Technologies); (B) fresh frozen tissues, displayed both with IGV from Broad Institute. Fresh frozen tissues $(B)$ showed an high coverage and an high read depth for exonic region compared to the FFPE samples $(A)$ performed with lon Total RNA-seq kit.

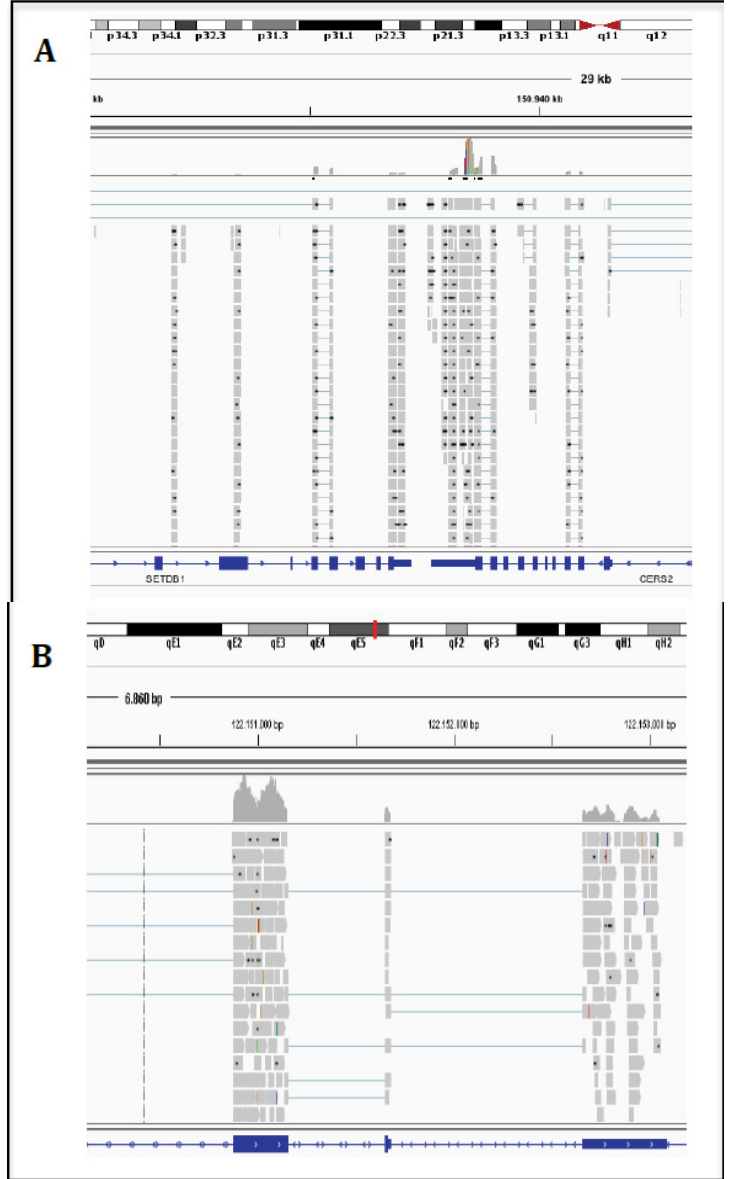

Figure 3: (A) FFPE LCM sample made with our modified protocol; (B) fresh frozen sample; (C) LCM fresh frozen sample and (D) FFPE sample displayed all with IGV. All the samples: FFPE (D),FFLPE LCM (A) and LCM fresh frozen (C) samples showed an high coverage and an high read depth in exonic regions, similar to fresh frozen samples $(A)$.

\begin{tabular}{|c|c|c|c|c|c|}
\hline Samples & Organism & Tissue type & $\begin{array}{l}\text { Reads Number } \\
\text { (aligned) }\end{array}$ & Coverage & Genes Covered ( $\geqq 20$ reads) \\
\hline $\begin{array}{c}\# 1 \\
9298\end{array}$ & Homo sapiens & Glioblastoma & 16857958 & $4 X$ & $13350 / 24137$ \\
\hline $\begin{array}{c}\# 2 \\
0303\end{array}$ & Homo sapiens & Glioblastoma & 36246545 & $10 x$ & $11084 / 24137$ \\
\hline $\begin{array}{c}\# 3 \\
2102\end{array}$ & Homo sapiens & Glioblastoma & 16262315 & $6 x$ & $7742 / 24137$ \\
\hline $\begin{array}{c}\# 4 \\
4096\end{array}$ & Homo sapiens & Glioblastoma & 28559434 & $9 x$ & $12434 / 24137$ \\
\hline $\begin{array}{c}\# 5 \\
4239\end{array}$ & Homo sapiens & Glioblastoma & 28114075 & $8 x$ & $16384 / 24137$ \\
\hline $\begin{array}{c}\# 6 \\
4318\end{array}$ & Homo sapiens & Glioblastoma & 78186377 & $20 x$ & $13837 / 24137$ \\
\hline $\begin{array}{c}\# 7 \\
4382\end{array}$ & Homo sapiens & Glioblastoma & 65755614 & $3 x$ & $2738 / 24137$ \\
\hline $\begin{array}{c}\# 8 \\
4534\end{array}$ & Homo sapiens & Glioblastoma & 14489319 & $5 X$ & $6227 / 24137$ \\
\hline $\begin{array}{c}\# 9 \\
4619\end{array}$ & Homo sapiens & Glioblastoma & 20319530 & $6 x$ & $9968 / 24137$ \\
\hline $\begin{array}{l}\# 10 \\
6043\end{array}$ & Homo sapiens & Glioblastoma & 15326390 & $4 X$ & $11928 / 24137$ \\
\hline $\begin{array}{l}\# 11 \\
7031\end{array}$ & Homo sapiens & Glioblastoma & 29771764 & $8 x$ & $13878 / 24137$ \\
\hline $\begin{array}{l}\# 12 \\
7901\end{array}$ & Homo sapiens & Glioblastoma & 30171079 & $9 x$ & $9681 / 24137$ \\
\hline
\end{tabular}


Citation: Lessi F, Aretini P, Franceschi S, La Ferla M, Negro S, et al. (2016) Whole Transcriptome Analysis with lon Torrent Platform on Challenging Tissues: Formalin-Fixed, Paraffin-Embedded Tissues and Laser Capture Microdissected Samples. Next Generat Sequenc \& Applic 3: 136. doi: 10.4172/2469-9853.1000136

Page 6 of 7

\begin{tabular}{|c|c|c|c|c|c|}
\hline $\begin{array}{c}\text { \# } 13 \\
\text { Insitu01 }\end{array}$ & Homo sapiens & Ductal breast carcinoma & 4966214 & $3 x$ & $10377 / 24137$ \\
\hline $\begin{array}{c}\text { \# } 14 \\
\text { Insitu03 }\end{array}$ & Homo sapiens & Ductal breast carcinoma & 6358112 & $5 X$ & $7037 / 24137$ \\
\hline $\begin{array}{c}\# 15 \\
\text { Inva01 }\end{array}$ & Homo sapiens & Ductal breast carcinoma & 10982961 & $6 X$ & $13453 / 24137$ \\
\hline $\begin{array}{c}\# 16 \\
\text { Norm01 }\end{array}$ & Homo sapiens & Ductal breast carcinoma & 7227120 & $4 X$ & $11994 / 24137$ \\
\hline $\begin{array}{c}\text { \# } 17 \\
\text { STQI01 }\end{array}$ & Homo sapiens & Ductal breast carcinoma & 5546112 & $3 X$ & $8178 / 24137$ \\
\hline $\begin{array}{c}\text { \# } 18 \\
\text { Inva03 }\end{array}$ & Homo sapiens & Ductal breast carcinoma & 4321973 & $3 X$ & $7295 / 24137$ \\
\hline $\begin{array}{c}\# 19 \\
\text { Inva04 }\end{array}$ & Homo sapiens & Ductal breast carcinoma & 9796789 & $15 X$ & $3299 / 24137$ \\
\hline $\begin{array}{c}\# 20 \\
\text { Inva05 }\end{array}$ & Homo sapiens & Ductal breast carcinoma & 15171934 & $19 x$ & $7367 / 24137$ \\
\hline $\begin{array}{c}\# 21 \\
\text { Norm03 }\end{array}$ & Homo sapiens & Ductal breast carcinoma & 3194111 & $3 X$ & $5315 / 24137$ \\
\hline $\begin{array}{c}\# 22 \\
\text { Norm05 }\end{array}$ & Homo sapiens & Ductal breast carcinoma & 27590579 & $29 x$ & $2032 / 24137$ \\
\hline $\begin{array}{c}\text { \# } 23 \\
\text { QINVA01 }\end{array}$ & Homo sapiens & Ductal breast carcinoma & 3751997 & $3 x$ & $9443 / 24137$ \\
\hline $\begin{array}{c}\text { \# } 24 \\
\text { QINVA03 }\end{array}$ & Homo sapiens & Ductal breast carcinoma & 4254834 & $3 x$ & $8170 / 24137$ \\
\hline $\begin{array}{c}\text { \# } 25 \\
\text { QINV04 }\end{array}$ & Homo sapiens & Ductal breast carcinoma & 11054499 & $11 \mathrm{X}$ & $4087 / 24137$ \\
\hline $\begin{array}{c}\# 26 \\
\text { SNO03 }\end{array}$ & Homo sapiens & Ductal breast carcinoma & 4348284 & $4 X$ & $1840 / 24137$ \\
\hline $\begin{array}{c}\text { \# } 27 \\
\text { QIN05 }\end{array}$ & Homo sapiens & Ductal breast carcinoma & 13291001 & $28 X$ & $1510 / 24137$ \\
\hline $\begin{array}{c}\text { \# } 28 \\
\text { InSi04 }\end{array}$ & Homo sapiens & Ductal breast carcinoma & 6367483 & $14 X$ & $3439 / 24137$ \\
\hline $\begin{array}{c}\# 29 \\
\text { InS05 }\end{array}$ & Homo sapiens & Ductal breast carcinoma & 22471493 & $23 X$ & $1920 / 24137$ \\
\hline $\begin{array}{c}\text { \#30 } \\
\text { Norm04 }\end{array}$ & Homo sapiens & Ductal breast carcinoma & 5804758 & $13 X$ & $2448 / 24137$ \\
\hline $\begin{array}{c}\text { \# } 31 \\
\text { STQI02 }\end{array}$ & Homo Sapiens & Ductal breast carcinoma & 1741379 & $2 X$ & $2146 / 24137$ \\
\hline $\begin{array}{c}\text { \# } 32 \\
\text { STQI04 }\end{array}$ & Homo sapiens & Ductal breast carcinoma & 5011860 & $17 X$ & $2775 / 24137$ \\
\hline $\begin{array}{c}\# 33 \\
4 \mathrm{H} 01\end{array}$ & Mus musculus & Neuromuscular junctions & 3546122 & $3 X$ & $6858 / 24460$ \\
\hline $\begin{array}{c}\# 34 \\
4 \mathrm{H}-02\end{array}$ & Mus musculus & Neuromuscular junctions & 4005529 & $4 X$ & $8559 / 24460$ \\
\hline $\begin{array}{c}\# 35 \\
4 \mathrm{H}-03\end{array}$ & Mus musculus & Neuromuscular junctions & 3354733 & $3 x$ & $7598 / 24460$ \\
\hline $\begin{array}{c}\# 36 \\
24 \mathrm{H} 01\end{array}$ & Mus musculus & Neuromuscolar junctions & 3314370 & $4 X$ & $4444 / 24460$ \\
\hline $\begin{array}{c}\# 37 \\
24 \mathrm{H} 02\end{array}$ & Mus musculus & Neuromuscolar junctions & 3515375 & $3 x$ & $11937 / 24460$ \\
\hline $\begin{array}{c}\text { \# } 38 \\
24 \mathrm{H} 03\end{array}$ & Mus musculus & Neuromuscolar junctions & 5195879 & $3 x$ & $9020 / 24460$ \\
\hline $\begin{array}{c}\text { \#39 } \\
96 \mathrm{H} 01\end{array}$ & Mus musculus & Neuromuscolar junctions & 2430560 & $3 x$ & $4653 / 24460$ \\
\hline $\begin{array}{c}\# 40 \\
96 \mathrm{H} 02\end{array}$ & Mus musculus & Neuromuscolar junctions & 2475209 & $3 x$ & $3746 / 24460$ \\
\hline $\begin{array}{c}\# 41 \\
96 \mathrm{H} 03\end{array}$ & Mus musculus & Neuromuscolar junctions & 4454109 & $6 X$ & $6209 / 24460$ \\
\hline $\begin{array}{c}\text { \# 42 } \\
\text { CTRL01 }\end{array}$ & Mus musculus & Neuromuscolar junctions & 2638485 & $3 x$ & $7736 / 24460$ \\
\hline $\begin{array}{c}\# 43 \\
\text { CTRL02 }\end{array}$ & Mus musculus & Neuromuscolar junctions & 3273245 & $3 x$ & $8529 / 24460$ \\
\hline $\begin{array}{c}\# 44 \\
\text { CTRL03 }\end{array}$ & Mus musculus & Neuromuscolar junctions & 3900189 & $3 x$ & $9574 / 24460$ \\
\hline $\begin{array}{c}\text { \# 45 } \\
\text { CTRL04I }\end{array}$ & Mus musculus & Neuromuscolar junctions & 4723034 & $61 X$ & $2255 / 24460$ \\
\hline
\end{tabular}

Table 2: Summary of the analyzed samples with the reads number, the coverage and the total of the covered genes. 
Citation: Lessi F, Aretini P, Franceschi S, La Ferla M, Negro S, et al. (2016) Whole Transcriptome Analysis with lon Torrent Platform on Challenging Tissues: Formalin-Fixed, Paraffin-Embedded Tissues and Laser Capture Microdissected Samples. Next Generat Sequenc \& Applic 3: 136. doi: 10.4172/2469-9853.1000136

Page 7 of 7

technique associated with the use of laser microdissection, allows to analyze specific regions of the tissues, also few cells achieving the whole gene expression. Eventually, but not less important, we stress that this application has never been done with the Ion Torrent platform.

\section{References}

1. Xu C, Houck JR, Fan W, Wang P, Chen Y, et al. (2008) Simultaneous isolation of DNA and RNA from the same cell population obtained by laser capture microdissection for genome and transcriptome profiling. J Mol Diagn 10: 129134.

2. Kohn AB, Moroz TP, Barnes JP, Netherton M, Moroz LL (2013) Single-Cell Semiconductor Sequencing. Methods Mol Biol 1048: 247-284.

3. Tang F, Lao K, Surani MA (2011) Development and applications of single-cell transcriptome analysis. Nat Methods 8: S6-11.

4. Tang F, Barbacioru C, Wang Y, Nordman E, Lee C, et al. (2009) mRNA-Seq whole-transcriptome analysis of a single cell. Nat Methods 6: 377-382.
5. Islam S, Kjällquist U, Moliner A, Zajac P, Fan JB (2011) Characterization of the single-cell transcriptional landscape by highly multiplex RNA-seq. Genome Res 21: 1160-1167.

6. Ramsköld D, Luo S, Wang YC, Li R, Deng Q, et al. (2012) Full-length mRNASeq from single-cell levels of RNA and individual circulating tumor cells. Nat Biotechnol 30: 777-782.

7. Tang F, Barbacioru C, Nordman E, Li B, Xu N, et al. (2010) RNA-Seq analysis to capture the transcriptome landscape of a single cell. Nat Protoc 5: 516-535.

8. Chenchik A, Zhu YY, Diatchenko L, Li R, Hill J, et al. (1998) Generation of highquality cDNA from small amounts of total RNA by SMART PCR. In: Siebert PD Larrick JW (Eds.) Gene Cloning and Analysis by RT-PCR. Eaton Publishing. Natick, MA, pp: 305-319.

9. Dobin A, Davis CA, Schlesinger F, Drenkow J, Zaleski C, et al. (2013) STAR ultrafast universal RNA-seq aligner. Bioinformatics 29: 15-21.

10. Lassmann T, Hayashizaki Y, Daub CO (2011) SAMStat: monitoring biases in next generation sequencing data. Bioinformatics 27: 130-131. 